\title{
Common fixed point theorems for
}

\section{Geraghty's type contraction mappings using the monotone property with two metrics}

\author{
Juan Martínez-Moreno ${ }^{1}$, Wutiphol Sintunavarat ${ }^{2^{*}}$ and Yeol Je Cho $\mathrm{C}^{3,4^{*}}$
}

"Correspondence:

wutiphol@mathstat.sci.tu.ac.th; yjcho@gnu.ac.kr

${ }^{2}$ Department of Mathematics and Statistics, Faculty of Science and Technology, Thammasat University Rangsit Center, Pathumthani, 12121, Thailand

${ }^{3}$ Department of Mathematics Education and the RINS,

Gyeongsang National University, Chinju, 660-701, Korea

Full list of author information is available at the end of the article

\begin{abstract}
The main aim of this paper is to obtain some new common fixed point theorems for Geraghty's type contraction mappings using the monotone property with two metrics and to give some examples to illustrate the main results. Further, by using our main results, we prove some results about multidimensional common fixed points. Our results generalize and extend some recent results given by Kadelburg et al. (Fixed Point Theory Appl. 2015:27, 2015) and Choudhurya and Kundu (J. Nonlinear Sci. Appl. 5:259-270, 2012).
\end{abstract}

Keywords: coincidence point; common fixed point; Geraghty's type contraction; mixed monotone property; partially ordered set

\section{Introduction and preliminaries}

Let $\Theta$ denote the class of real functions $\theta:[0, \infty) \rightarrow[0,1)$ satisfying the following condition:

$$
\theta\left(t_{n}\right) \rightarrow 1 \quad \Longrightarrow \quad t_{n} \rightarrow 0 .
$$

An example of a function in $\Theta$ may be given by $\theta(t)=e^{-2 t}$ for all $t>0$ and $\theta(0) \in[0,1)$. In 1973, Geraghty [1] proved the following theorem, which is a generalization of Banach's contraction principle:

Theorem 1.1 ([1]) Let $(X, d)$ be a complete metric space and $f: X \rightarrow X$ be a self-mapping. Suppose that there exists $\theta \in \Theta$ such that

$$
d(f x, f y) \leq \theta(d(x, y)) d(x, y)
$$

for all $x, y \in X$. Then $f$ has a unique fixed point in $X$.

Recently, Amini-Harandi and Emami [2] extended this result to the setting of partially ordered metric spaces as follows:

Theorem 1.2 ([2]) Let $(X, d)$ be a complete partially ordered metric space and $f: X \rightarrow X$ be an increasing self-mapping such that there exists $x_{0} \in X$ such that $x_{0} \preceq f x_{0}$. Suppose that 
there exists $\theta \in \Theta$ such that

$$
d(f x, f y) \leq \theta(d(x, y)) d(x, y)
$$

for all $x, y \in X$ satisfying $x \preceq y$ or $x \succeq y$. Then, in each of the following two cases, the mapping $f$ has at least one fixed point in $X$ :

(1) $f$ is continuous or

(2) for any non-decreasing sequence $\left\{x_{n}\right\}$ in $X$, if $x_{n} \rightarrow x \in X$ as $n \rightarrow \infty$, then $x_{n} \preceq x$ for all $n \geq 1$.

If, moreover, for all $x, y \in X$, there exists $z \in X$ comparable with $x$ and $y$, then the fixed point off is unique.

For more generalizations of Theorems 1.1 and 1.2, see [3-5].

On the other hand, several authors have studied fixed point theory in spaces equipped with two metrics (see [6-8]). Especially, Agarwal and O'Regan [6] proved the following:

Let $\left(X, d^{\prime}\right)$ be a metric space and $d$ be another metric on $X$. For any $x_{0} \in X$ and $r>0$, let

$$
B\left(x_{0}, r\right)=\left\{x \in X: d\left(x, x_{0}\right)<r\right\}
$$

and let $\overline{B\left(x_{0}, r\right)^{d \prime}}$ denote the $d^{\prime}$-closure of $B\left(x_{0}, r\right)$.

Theorem 1.3 ([6]) Let $\left(X, d^{\prime}\right)$ be a complete metric space, $d$ be another metric on $X, x_{0} \in X$, $r>0$, and $F: \overline{B\left(x_{0}, r\right)^{d \prime}} \rightarrow X$ be a mapping. Suppose that there exists $q \in(0,1)$ such that, for all $x, y \in \overline{B\left(x_{0}, r\right)^{d \prime}}$,

$$
d(F x, F y) \leq q \max \left\{d(x, y), d(x, F x), d(y, F y), \frac{1}{2}[d(x, F y)+d(y, F x)]\right\} .
$$

In addition, assume that the following three properties hold:

(1) $d\left(x_{0}, F x_{0}\right)<(1-q) r$;

(2) if $d \ngtr d^{\prime}$, assume that $F$ is uniformly continuous from $\left(B\left(x_{0}, r\right), d\right)$ into $\left(X, d^{\prime}\right)$;

(3) if $d \neq d^{\prime}$, assume that $F$ is continuous from $\left(\overline{B\left(x_{0}, r\right)^{d^{\prime}}}, d\right)$ into $\left(X, d^{\prime}\right)$.

Then $F$ has a fixed point, that is, there exists $x \in \overline{B\left(x_{0}, r\right)^{d \prime}}$ with $x=F x$.

The aim of this paper is to study some new common fixed point theorems for Geraghty's type contraction mappings using the monotone property with two metrics, which is an important advantage to compare with well known fixed point theorems in metric spaces. Further, we give some examples to illustrate the main results. The main results in this paper generalize, unify, and extend some recent results given by some authors.

\section{Main results}

In this section, we prove some fixed point results for generalized contractions on spaces with two metrics.

Throughout this paper, $(X, \preceq)$ denotes a partially ordered set. By $x \succeq y$, we mean $y \preceq x$. Let $f, g: X \rightarrow X$ be two mappings. A mapping $f$ is said to be $g$-non-decreasing (resp., $g$ non-increasing) if, for all $x, y \in X, g x \preceq g y$ implies $f x \preceq f y$ (resp., $f y \preceq f x$ ). If $g$ is the identity 
mapping, then $f$ is said to be non-decreasing (resp., non-increasing). Let $d^{\prime}, d$ be two metrics on $X$. By $d<d^{\prime}$ (resp., $d \leq d^{\prime}$ ), we mean $d(x, y)<d^{\prime}(x, y)$ (resp., $d(x, y) \leq d^{\prime}(x, y)$ ) for all $x, y \in X$.

Also, we give some essential concepts which are useful for our main results:

Definition 2.1 ([9]) Let $(X, d)$ be a metric space and $f, g: X \rightarrow X$ be two mappings. The mappings $g$ and $f$ are said to be $d$-compatible if

$$
\lim _{n \rightarrow \infty} d\left(g f x_{n}, f g x_{n}\right)=0
$$

whenever $\left\{x_{n}\right\}$ is sequences in $X$ such that $\lim _{n \rightarrow \infty} f x_{n}=\lim _{n \rightarrow \infty} g x_{n}$.

Definition 2.2 ([9]) Let $(X, d)$ and $\left(Y, d^{\prime}\right)$ be two metric spaces and $f: X \rightarrow Y$ and $g: X \rightarrow$ $X$ be two mappings. A mapping $f$ is said to be $g$-uniformly continuous on $X$ if, for any real number $\epsilon>0$, there exists $\delta>0$ such that $d^{\prime}(f x, f y)<\epsilon$ whenever $x, y \in X$ and $d(g x, g y)<\delta$. If $g$ is the identity mapping, then $f$ is said to be uniformly continuous on $X$.

Now, we give the main result in this paper.

Theorem 2.3 Let $\left(X, d^{\prime}, \preceq\right)$ be a complete partially ordered metric space, $d$ be another metric on $X$ and $g, f: X \rightarrow X$ be two mappings such that $f$ has the $g$-monotone property. Suppose that the following conditions hold:

(1) $g:\left(X, d^{\prime}\right) \rightarrow\left(X, d^{\prime}\right)$ is continuous and $g(X)$ is $d^{\prime}$-closed;

(2) $f(X) \subseteq g(X)$;

(3) there exists $x_{0} \in X$ such that $g x_{0} \preceq f x_{0}$;

(4) there exists $\theta \in \Theta$ such that

$$
d(f x, f y) \leq \theta(d(g x, g y)) d(g x, g y)
$$

for all $x, y \in X$ with $g x \leq$ gy or $g x \geq g y$ :

(5) if $d \nsupseteq d^{\prime}$, assume that $f:(X, d) \rightarrow\left(X, d^{\prime}\right)$ is g-uniformly continuous;

(6) if $d \neq d^{\prime}$, assume that $f:\left(X, d^{\prime}\right) \rightarrow\left(X, d^{\prime}\right)$ is continuous and $g$ and $f$ are $d^{\prime}$-compatible;

(7) if $d=d^{\prime}$, assume that (a) $f$ is continuous and $g$ and $f$ are compatible or (b) for any non-decreasing sequence $\left\{x_{n}\right\}$ in $X$, if $x_{n} \rightarrow x \in X$ as $n \rightarrow \infty$, then $x_{n} \preceq x$ for all $n \geq 1$. Then there exists $u \in X$ such that $g u=f u$, i.e., $g$ and $f$ have a coincidence point.

Proof Starting from $x_{0}$ (the condition (3)) and using $f(X) \subseteq g(X)$ (the condition (2)), we can construct a sequence $\left\{x_{n}\right\}$ in $X$ such that

$$
g x_{n}=f x_{n-1}
$$

for all $n \in \mathbb{N}$. If $g x_{n_{0}}=g x_{n_{0}-1}$ for some $n_{0} \in \mathbb{N}$, then $x_{n_{0}-1}$ is a coincidence point of the mappings $g$ and $f$. Therefore, we assume that, for each $n \in \mathbb{N}, g x_{n} \neq g x_{n-1}$ holds.

By the condition (3), $g x_{0} \preceq f x_{0}=g x_{1}$ and so the $g$-monotone property of $F$ implies that $g x_{1}=f x_{0} \preceq f x_{1}=g x_{2}$. Proceeding by induction, we have $g x_{n-1} \preceq g x_{n}$ for each $n \in \mathbb{N}$. Hence 
it follows from the contractive condition (2.1) that

$$
\begin{aligned}
d\left(g x_{n}, g x_{n+1}\right) & =d\left(f x_{n-1}, f x_{n}\right) \\
& \leq \theta\left(d\left(g x_{n-1}, g x_{n}\right)\right) d\left(g x_{n-1}, g x_{n}\right) \\
& <d\left(g x_{n-1}, g x_{n}\right)
\end{aligned}
$$

for all $n \in \mathbb{N}$. Thus the sequence $\left\{d_{n}\right\}:=\left\{d\left(g x_{n-1}, g x_{n}\right)\right\}$ is decreasing and so it follows that $d_{n} \rightarrow \alpha$ as $n \rightarrow \infty$ for some $\alpha \geq 0$.

Next, we claim that $\alpha=0$. Assume on the contrary that $\alpha>0$. Then it follows from (2.2) that

$$
\frac{d_{n+1}}{d_{n}} \leq \theta\left(d_{n}\right)<1
$$

Letting $n \rightarrow \infty$, we get $\theta\left(d_{n}\right) \rightarrow 1$ as $n \rightarrow \infty$. Since $\theta \in \Theta$, we have $d_{n} \rightarrow 0$ as $n \rightarrow$ $\infty$, which contradicts the assumption $\alpha>0$. Therefore, we can conclude that $d_{n}=$ $d\left(g x_{n-1}, g x_{n}\right) \rightarrow 0$ as $n \rightarrow \infty$.

Now, we show that $\left\{g x_{n}\right\}$ is a Cauchy sequence with respect to $d$. Suppose that $\left\{g x_{n}\right\}$ is not a Cauchy sequence with respect to $d$. Then there exists $\epsilon>0$ for which we can find subsequences $\left\{g x_{n_{k}}\right\},\left\{g x_{m_{k}}\right\}$ of $\left\{g x_{n}\right\}$ such that $n_{k}>m_{k} \geq k$ satisfying

$$
d\left(g x_{n_{k}}, g x_{m_{k}}\right) \geq \epsilon, \quad d\left(g x_{n_{k}-1}, g x_{m_{k}}\right)<\epsilon
$$

Using (2.3) and the triangle inequality, we have

$$
\begin{aligned}
\epsilon & \leq r_{k}:=d\left(g x_{n_{k}}, g x_{m_{k}}\right) \\
& \leq d\left(g x_{n_{k}}, g x_{n_{k}-1}\right)+d\left(g x_{n_{k}-1}, g x_{m_{k}}\right) \\
& <d\left(g x_{n_{k}}, g x_{n_{k}-1}\right)+\epsilon .
\end{aligned}
$$

Letting $k \rightarrow \infty$, we have

$$
r_{k}=d\left(g x_{n_{k}}, g x_{m_{k}}\right) \rightarrow \epsilon
$$

Again, by the triangle inequality and the contractive condition (2.1), we have

$$
\begin{aligned}
r_{k} & =d\left(g x_{n_{k}}, g x_{m_{k}}\right) \\
& \leq d\left(g x_{n_{k}}, g x_{n_{k}+1}\right)+d\left(g x_{n_{k}+1}, g x_{m_{k}+1}\right)+d\left(g x_{m_{k}+1}, g x_{m_{k}}\right) \\
& =d\left(g x_{n_{k}}, g x_{n_{k}+1}\right)+d\left(g x_{m_{k}+1}, g x_{m_{k}}\right)+d\left(f x_{n_{k}}, f x_{m_{k}}\right) \\
& \leq d\left(g x_{n_{k}}, g x_{n_{k}+1}\right)+d\left(g x_{m_{k}+1}, g x_{m_{k}}\right)+\theta\left(d\left(g x_{n_{k}}, g x_{m_{k}}\right)\right) d\left(g x_{n_{k}}, g x_{m_{k}}\right) \\
& =d_{n_{k}+1}+d_{m_{k}+1}+\theta\left(r_{k}\right) r_{k} \\
& <d_{n_{k}+1}+d_{m_{k}+1}+r_{k} .
\end{aligned}
$$

Now, we have

$$
r_{k} \leq d_{n_{k}+1}+d_{m_{k}+1}+\theta\left(r_{k}\right) r_{k}<d_{n_{k}+1}+d_{m_{k}+1}+r_{k}
$$


Letting $k \rightarrow \infty$ and using (2.4), we have $\theta\left(r_{k}\right) \rightarrow 1$ and so, using the properties of function $\theta$, we obtain $r_{k} \rightarrow 0$ as $k \rightarrow \infty$, which contradicts $\epsilon>0$. Therefore, it follows that $\left\{g x_{n}\right\}$ is a Cauchy sequence respect to $d$.

Also, we claim that $\left\{g x_{n}\right\}$ is a Cauchy sequence with respect to $d^{\prime}$. If $d \geq d^{\prime}$, it is trivial. Thus, suppose $d \nsupseteq d^{\prime}$. Let $\bar{\epsilon}>0$ be given. Now, the condition (5) guarantees that there exists $\delta$ such that

$$
d^{\prime}(f x, f y)<\bar{\epsilon}
$$

whenever $x, y \in X$ and $d(g x, g y)<\delta$. Since $\left\{g x_{n}\right\}$ is a Cauchy sequence respect to $d$, there exists $n_{0} \in \mathbb{N}$ with

$$
d\left(g x_{n}, g x_{m}\right)<\delta
$$

whenever $n, m \geq n_{0}$. Now, (2.5) and (2.6) imply that

$$
d^{\prime}\left(g x_{n+1}, g x_{m+1}\right)=d^{\prime}\left(f x_{n}, f x_{m}\right)<\bar{\epsilon}
$$

whenever $n, m \geq n_{0}$ and so $\left\{g x_{n}\right\}$ is a Cauchy sequence respect to $d^{\prime}$. Since $g(X)$ is a $d^{\prime}$ closed subset of the complete metric space $\left(X, d^{\prime}\right)$, there exists $u=g x \in g(X)$ such that

$$
\lim _{n \rightarrow \infty} g x_{n}=\lim _{n \rightarrow \infty} f x_{n}=u .
$$

Finally, we prove that $u$ is a common fixed point of $f$ and $g$. We consider two cases:

Case I: $d \neq d^{\prime}$.

By the $d^{\prime}$-compatibility of $g$ and $f$, we have

$$
\lim _{n \rightarrow \infty} d^{\prime}\left(g f x_{n}, f g x_{n}\right)=0 .
$$

Using the triangle inequality, we have

$$
d^{\prime}\left(g u, f g x_{n}\right) \leq d^{\prime}\left(g u, g f x_{n}\right)+d^{\prime}\left(g f x_{n}, f g x_{n}\right) .
$$

Letting $n \rightarrow \infty$, from (2.7) and the continuity of $g$ and $f$, it follows that $d^{\prime}(g u, f u)=0$, i.e., $g u=f u$.

Case II: $d=d^{\prime}$.

In order to avoid the repetition, we can only consider (b) of the condition (7). In this case, there exists $x \in X$ such that $g x_{n} \preceq u=g x$ for each $n \in \mathbb{N}$. Using (2.1), we have

$$
\begin{aligned}
d(f x, g x) & \leq d\left(f x, g x_{n+1}\right)+d\left(g x_{n+1}, g x\right) \\
& =d\left(f x, f x_{n}\right)+d\left(g x_{n+1}, g x\right) \\
& \leq \theta\left(d\left(g x, g x_{n}\right)\right) d\left(g x, g x_{n}\right)+d\left(g x_{n+1}, g x\right) \\
& <d\left(g x, g x_{n}\right)+d\left(g x_{n+1}, g x\right) \rightarrow 0
\end{aligned}
$$

as $n \rightarrow \infty$. Hence $g x=f x$. This completes the proof.

Now, we give some examples to illustrate Theorem 2.3. 
Example 2.4 Let $X=[0, \infty) \subseteq \mathbb{R}$ and the metrics $d, d^{\prime}: X \times X \rightarrow[0, \infty)$ be defined by

$$
d(x, y)=|x-y|, \quad d^{\prime}(x, y)=L|x-y|
$$

for all $x, y \in X$, respectively, where $L$ is a constant real number such that $L \in(1, \infty)$. It is easy to see that $d<d^{\prime}$.

Now, we consider the partially order $\preceq$ in $X$ given by

$$
x \preceq y \quad \Longleftrightarrow \quad x=y \text { or }[x, y \in\{1 / n: n \in \mathbb{N}\} \cup\{0\} \text { with } x \leq y],
$$

where $\leq$ is the usual order. Consider the mappings $f: X \rightarrow X$ and $g: X \rightarrow X$ defined by

$$
g x=x^{2}, \quad f x=\ln \left(1+\frac{x^{2}}{2}\right)
$$

for all $x \in X$, respectively. By using the increasing property of the function logarithm, we see that $f$ has the $g$-monotone property.

Next, we show that the conditions (1)-(7) in Theorem 2.3 hold as follows:

(1) We can easily check that $g:\left(X, d^{\prime}\right) \rightarrow\left(X, d^{\prime}\right)$ is continuous. Also, we can see that $g(X)=[0, \infty)$ is $d^{\prime}$-closed.

(2) By the definition of $f$ and $g$, we can see that $f(X)=g(X)$.

(3) It is easy to see that there exists a point $x_{0} \in X$ such that $g x_{0} \preceq f x_{0}$.

(4) Let $\theta \in \Theta$ be defined by

$$
\theta(t)= \begin{cases}\frac{2 \ln \left(1+\frac{t}{2}\right)}{t}, & \text { if } t>0 \\ 0, & \text { if } t=0\end{cases}
$$

Let $x, y$ be arbitrary points in $X$ and suppose that $g x \preceq g y$. If $g x=g y$, we have $x=y$ and hence the contractive condition (2.1) holds for this case. In another case, we have

$$
g x, g y \in\{1 / n: n \in \mathbb{N}\} \cup\{0\} \text { with } g x \leq g y .
$$

Then we obtain $g x=x^{2}, g y=y^{2} \in[0,1]$, and $x^{2}=g x \leq g y=y^{2}$. Also, we have

$$
\begin{aligned}
d(f x, f y) & =\left|\ln \left(1+\frac{x^{2}}{2}\right)-\ln \left(1+\frac{y^{2}}{2}\right)\right| \\
& =\ln \left(1+\frac{y^{2}}{2}\right)-\ln \left(1+\frac{x^{2}}{2}\right) \\
& =\ln \frac{1+\frac{y^{2}}{2}}{1+\frac{x^{2}}{2}} \\
& =\ln \left(1+\frac{\frac{y^{2}}{2}-\frac{x^{2}}{2}}{1+\frac{x^{2}}{2}}\right) \\
& \leq \ln \left(1+\left|\frac{x^{2}}{2}-\frac{y^{2}}{2}\right|\right)
\end{aligned}
$$




$$
\begin{aligned}
& \leq \frac{2 \ln \left(1+\frac{1}{2}\left|x^{2}-y^{2}\right|\right)}{\left|x^{2}-y^{2}\right|}\left|x^{2}-y^{2}\right| \\
& =\frac{2 \ln \left(1+\frac{1}{2} d(g x, g y)\right)}{d(g x, g y)} d(g x, g y) \\
& =\theta(d(g x, g y)) d(g x, g y) .
\end{aligned}
$$

Similarly, we can also prove that the condition (2.1) holds for case of $g x \geq g y$. Therefore, the condition (4) holds with the function $\theta$.

(5) Since $d<d^{\prime}$, we show that a mapping $f:(X, d) \rightarrow\left(X, d^{\prime}\right)$ is $g$-uniformly continuous. Let $\epsilon>0$ be given and choose $\delta:=\frac{\epsilon}{L}$. Assume that $x, y \in X$ with $d(g x, g y)<\delta=\frac{\epsilon}{L}$. Then we have

$$
\begin{aligned}
d^{\prime}(f x, f y) & =L|f x-f y| \\
& =L\left|\ln \left(1+\frac{x^{2}}{2}\right)-\ln \left(1+\frac{y^{2}}{2}\right)\right| \\
& =L\left|\ln \frac{1+\frac{y^{2}}{2}}{1+\frac{x^{2}}{2}}\right| \\
& =L\left|\ln \left(1+\frac{\frac{y^{2}}{2}-\frac{x^{2}}{2}}{1+\frac{x^{2}}{2}}\right)\right| \\
& \leq L\left[\ln \left(1+\left|\frac{x^{2}}{2}-\frac{y^{2}}{2}\right|\right)\right] \\
& \leq L\left[\frac{2 \ln \left(1+\frac{1}{2}\left|x^{2}-y^{2}\right|\right)}{\left|x^{2}-y^{2}\right|}\left|x^{2}-y^{2}\right|\right] \\
& <L\left|x^{2}-y^{2}\right| \\
& =L d(g x, g y) \\
& <L \frac{\epsilon}{L} \\
& =\epsilon .
\end{aligned}
$$

This implies that $f:(X, d) \rightarrow\left(X, d^{\prime}\right)$ is $g$-uniformly continuous.

(6) Since $d \neq d^{\prime}$, we prove that $f:\left(X, d^{\prime}\right) \rightarrow\left(X, d^{\prime}\right)$ is continuous and $g$ and $f$ are $d^{\prime}$ compatible. It is easy to see that $f:\left(X, d^{\prime}\right) \rightarrow\left(X, d^{\prime}\right)$ is continuous. So we will only show that $g$ and $f$ are $d^{\prime}$-compatible. Let $\left\{x_{n}\right\}$ be a sequence in $X$ such that

$$
\lim _{n \rightarrow \infty} g x_{n}=\lim _{n \rightarrow \infty} f x_{n}=a .
$$

Then we obtain $\ln \left(1+\frac{a}{2}\right)=a$ and so it follows that $a=0$. Now, we have

$$
d^{\prime}\left(g f x_{n}, f g x_{n}\right)=L\left|\left(\ln \left(1+\frac{x_{n}^{2}}{2}\right)\right)^{2}-\ln \left(1+\frac{x_{n}^{4}}{2}\right)\right| \rightarrow 0
$$

as $n \rightarrow \infty$.

(7) Since $d \neq d^{\prime}$, we have nothing to do to show this condition. 
Consequently, all the conditions of Theorem 2.3 hold. Therefore, $g$ and $f$ have a coincidence point and, further, a point 0 is a coincidence point of the mappings $g$ and $f$.

Example 2.5 Let $X=[0, \infty) \subseteq \mathbb{R}$ and the metrics $d, d^{\prime}: X \times X \rightarrow[0, \infty)$ be defined by

$$
d(x, y)= \begin{cases}0, & \text { if } x=y \\ \max \{x, y\}, & \text { if } x \neq y\end{cases}
$$

and

$$
d^{\prime}(x, y)=|x-y|
$$

for all $x, y \in X$, respectively. It is easy to see that $d \geq d^{\prime}$.

Now, we consider the partially order $\preceq$ in $X$ given by

$$
x \preceq y \quad \Longleftrightarrow \quad x=y \text { or }[x, y \in[0,1 / 16] \text { with } x \leq y] \text {, }
$$

where $\leq$ is the usual order. Consider the mappings $f: X \rightarrow X$ and $g: X \rightarrow X$ defined by

$$
g x=x^{4}, \quad f x=x^{6}
$$

for all $x \in X$, respectively. It is easy to see that $f$ has the $g$-monotone property.

Next, we show that the conditions (1)-(7) in Theorem 2.3 hold as follows:

(1) We can easily check that $g:\left(X, d^{\prime}\right) \rightarrow\left(X, d^{\prime}\right)$ is continuous. Also, we can see that $g(X)=[0, \infty)$ is $d^{\prime}$-closed.

(2) By the definition of $f$ and $g$, we can see that $f(X)=g(X)$.

(3) It is easy to see that there exists a point $x_{0} \in X$ such that $g x_{0} \preceq f x_{0}$.

(4) Let $\theta \in \Theta$ be defined by

$$
\theta(t)= \begin{cases}\frac{1}{4}, & \text { if } 0 \leq t<1, \\ t^{2}+2, & \text { if } t \geq 1\end{cases}
$$

Let $x, y$ be arbitrary points in $X$ and suppose that $g x \preceq g y$. If $g x=g y$, we have $x=y$ and hence the contractive condition (2.1) holds for this case. In another case, we have

$$
g x=x^{4}, \quad g y=y^{4} \in[0,1 / 16] \text { with } g x \leq g y .
$$

Then we obtain $x, y \in[0,1 / 2]$ and $x \leq y$. Also, we have

$$
\begin{aligned}
d(f x, f y) & =\max \left\{x^{6}, y^{6}\right\} \\
& =y^{6} \\
& \leq \frac{1}{4} y^{4} \\
& =\theta\left(y^{4}\right) y^{4} \\
& =\theta\left(\max \left\{x^{4}, y^{4}\right\}\right) \max \left\{x^{4}, y^{4}\right\} \\
& =\theta(d(g x, g y)) d(g x, g y) .
\end{aligned}
$$


Similarly, we can also prove that the condition (2.1) holds for case of $g x \geq g y$. Therefore, the condition (4) holds with the function $\theta$.

(5) It follows from $d \geq d^{\prime}$ that we have nothing to do to show this condition.

(6) Since $d \neq d^{\prime}$, we will prove that $f:\left(X, d^{\prime}\right) \rightarrow\left(X, d^{\prime}\right)$ is continuous and $g$ and $f$ are $d^{\prime}$-compatible. It is easy to see that $f:\left(X, d^{\prime}\right) \rightarrow\left(X, d^{\prime}\right)$ is continuous. So we will only show that $g$ and $f$ are $d^{\prime}$-compatible. Suppose that $\left\{x_{n}\right\}$ is a sequence in $X$ such that

$$
\lim _{n \rightarrow \infty} g x_{n}=\lim _{n \rightarrow \infty} f x_{n}=a .
$$

for some $a \in X$. Now, we have

$$
d^{\prime}\left(g f x_{n}, f g x_{n}\right)=\left|x_{n}^{24}-x_{n}^{24}\right|=0
$$

for all $n \in \mathbb{N}$. This implies that $d^{\prime}\left(g f x_{n}, f g x_{n}\right) \rightarrow 0$ as $n \rightarrow \infty$.

(7) Since $d \neq d^{\prime}$, we have nothing to do to show this condition.

Consequently, all the conditions of Theorem 2.3 hold. Therefore, $g$ and $f$ have a coincidence point and, further, the points 0 and 1 are coincidence points of the mappings $g$ and $f$.

Putting $g=I_{X}$, where $I_{X}$ is the identity mapping on $X$ in Theorem 2.3, we obtain the following:

Corollary 2.6 Let $\left(X, d^{\prime}, \preceq\right)$ be a complete partially ordered metric space, $d$ be another metric on $X$ and $f: X \rightarrow X$ be a monotone mapping. Suppose that the following hold:

(1) there exists $x_{0} \in X$ such that $x_{0} \preceq f x_{0}$;

(2) there exists $\theta \in \Theta$ such that

$$
d(f x, f y) \leq \theta(d(x, y)) d(x, y)
$$

for all $x, y \in X$ with $x \preceq y$ or $x \succeq y$;

(3) if $d \ngtr d^{\prime}$, assume that $f:(X, d) \rightarrow\left(X, d^{\prime}\right)$ is uniformly continuous;

(4) if $d \neq d^{\prime}$, assume that $f:(X, d) \rightarrow(X, d)$ is continuous;

(5) if $d=d^{\prime}$, then (a) $f$ is continuous or (b) for any non-decreasing sequence $\left\{x_{n}\right\}$ in $X$, if $x_{n} \rightarrow x \in X$ as $n \rightarrow \infty$, then $x_{n} \preceq x$ for all $n$.

Then there exists $u \in X$ such that $u=f u$, i.e., $f$ has a fixed point.

Taking $d=d^{\prime}$ in Theorem 2.3, we have the following:

Theorem 2.7 Let $(X, d, \preceq)$ be a complete partially ordered metric space and $g: X \rightarrow X$, $f: X \rightarrow X$ be two mappings such that $f$ has the g-monotone property. Suppose that the following hold:

(1) $g$ is continuous and $g(X)$ is closed;

(2) $f(X) \subseteq g(X)$;

(3) there exists $x_{0} \in X$ such that $g x_{0} \preceq f x_{0}$; 
(4) there exists $\theta \in \Theta$ such that

$$
d(f x, f y) \leq \theta(d(g x, g y)) d(g x, g y)
$$

for all $x, y \in X$ with $g x \preceq$ gy or $g x \geq g y$;

(5) (a) $f$ is continuous and $g$ and $f$ are compatible or (b) for any non-decreasing sequence $\left\{x_{n}\right\}$ in $X$, if $x_{n} \rightarrow x \in X$ as $n \rightarrow \infty$, then $x_{n} \preceq x$ for all $n$.

Then there exists $u \in X$ such that $g u=f u$, i.e., $g$ and $f$ have a coincidence point.

Theorem 2.8 In addition to the hypotheses of Theorem 2.3, assume that

(8) for any $x, u \in X$, there exists $y \in X$ such that fy is comparable to both $f x$ and $f u$.

If $f$ and $g$ are $d^{\prime}$-compatible, then $g$ and $f$ have a common fixed point, i.e., there exists a point $p \in X$ such that $p=g p=f p$.

Proof Theorem 2.3 implies that there exists a coincidence point $x \in X$, that is, $g x=f x$. Suppose that there exists another coincidence point $u \in X$ and hence $g u=f u$.

Now, we prove that $g x=g u$. In fact, from the condition (8), it follows that there exists $y \in X$ such that $f y$ is comparable to both $f x$ and $f u$. Put $y_{0}=y$ and, analogously to the proof of Theorem 2.3, choose a sequence $\left\{y_{n}\right\}$ in $X$ satisfying

$$
g y_{n}=f y_{n-1}
$$

for all $n \in \mathbb{N}$. Starting from $x_{0}=x$ and $u_{0}=u$, choose the sequences $\left\{x_{n}\right\}$ and $\left\{u_{n}\right\}$ satisfying $g x_{n}=f x_{n-1}$ and $g u_{n}=f u_{n-1}$ for each $n \in \mathbb{N}$. Taking into account the properties of coincidence points, it is easy to see that it can be done so that $x_{n}=x$ and $u_{n}=u$, i.e.,

$$
g x_{n}=f x, \quad g u_{n}=f u
$$

for all $n \in \mathbb{N}$. Since $f x=g x$ and $f y=g y_{1}$ are comparable, then $g x \preceq g y_{n}$ or $g y_{n} \preceq g x$ for all $n \in \mathbb{N}$. Thus we can apply the contractive condition (2.1) to obtain

$$
d\left(g x, g y_{n+1}\right)=d\left(f x, f y_{n}\right) \leq \theta\left(d\left(g x, g y_{n}\right)\right) d\left(g x, g y_{n}\right)<d\left(g x, g y_{n}\right)
$$

for all $n \in \mathbb{N}$. Therefore, we can show that the sequence $\left\{d_{n}\right\}:=\left\{d\left(g x, g y_{n}\right)\right\}$ is decreasing and hence $d_{n} \rightarrow \alpha$ as $n \rightarrow \infty$ for some $\alpha \geq 0$.

Now, we prove that $\alpha=0$. Assume that $\alpha>0$. Then it follows from (2.10) that

$$
\frac{d_{n+1}}{d_{n}} \leq \theta\left(d_{n}\right)<1
$$

Letting $n \rightarrow \infty$ in the above inequality, we have $\theta\left(d_{n}\right) \rightarrow 1$ as $n \rightarrow \infty$. By the property $\left(\theta_{2}\right)$ of $\theta \in \Theta$, we have $d_{n} \rightarrow 0$ as $n \rightarrow \infty$, which contradicts the assumption $\alpha>0$. Therefore, we can conclude that $d\left(g x, g y_{n}\right) \rightarrow 0$ as $n \rightarrow \infty$. Similarly, we can prove that $d\left(g u, g y_{n}\right) \rightarrow 0$ as $n \rightarrow \infty$. By the triangle inequality, we have

$$
d(g x, g u) \leq d\left(g x, g y_{n}\right)+d\left(g y_{n}, g u\right)
$$


for all $n \in \mathbb{N}$. Letting $n \rightarrow \infty$ in the above inequality, it follows that $d(g x, g u)=0$. Therefore, we have $g x=g u$.

Now, let $p:=g x$. Hence we have $g p=g g x=g f x$. By the definition of the sequence $\left\{x_{n}\right\}$, we have $g x_{n}=f x=f x_{n-1}$ for all $n \in \mathbb{N}$ and so

$$
\lim _{n \rightarrow \infty} f x_{n}=\lim _{n \rightarrow \infty} g x_{n}=f x
$$

with respect to $d^{\prime}$. Since $g$ and $f$ are $d^{\prime}$-compatible, we have

$$
\lim _{n \rightarrow \infty} d^{\prime}\left(g f x_{n}, f g x_{n}\right)=0
$$

that is, $g f x=f g x$. Therefore, we have $g p=g f x=f g x=f p$. This implies that $p$ is another coincidence point of the mappings $f$ and $g$. By the property we have just proved, it follows that $f p=g p=g x=p$ and so $p$ is a common fixed point of $g$ and $f$. This completes the proof.

\section{Some particular cases}

First, we give some definitions for the main results in this section.

Definition 3.1 Let $(X, \preceq)$ be a partially ordered set and $F: X \times X \rightarrow X, g: X \rightarrow X$ be two mappings. The mapping $F$ is said to have the $g$-monotone property if $F$ is monotone $g$-non-decreasing in both of its arguments, that is, for any $x, y \in X$,

$$
x_{1}, x_{2} \in X, \quad g x_{1} \preceq g x_{2} \quad \Longrightarrow \quad F\left(x_{1}, y\right) \preceq F\left(x_{2}, y\right)
$$

and

$$
y_{1}, y_{2} \in X, \quad g y_{1} \preceq g y_{2} \quad \Longrightarrow \quad F\left(x, y_{1}\right) \preceq F\left(x, y_{2}\right) .
$$

If, in the previous relations, $g$ is the identity mapping, then $F$ is said to have the monotone property.

Definition 3.2 ([10,11]) Let $X$ be a nonempty set and $F: X \times X \rightarrow X, g: X \rightarrow X$ be two mappings. An element $(x, y) \in X \times X$ is called:

$\left(C_{1}\right)$ a coupled fixed point of $F$ if $x=F(x, y)$ and $y=F(y, x)$;

$\left(\mathrm{C}_{2}\right)$ a coupled coincidence point of $g$ and $F$ if $g x=F(x, y)$ and $g y=F(y, x)$ and, in this case, a point $(g x, g y)$ is called a coupled point of coincidence;

$\left(\mathrm{C}_{3}\right)$ a common coupled fixed point of $g$ and $F$ if $x=g x=F(x, y)$ and $y=g y=F(y, x)$.

Definition 3.3 ([12]) Let $(X, d)$ be a metric space. Two mappings $g: X \rightarrow X$ and $F: X \times$ $X \rightarrow X$ are said to be $d$-compatible if

$$
\lim _{n \rightarrow \infty} d\left(g F\left(x_{n}, y_{n}\right), F\left(g x_{n}, g y_{n}\right)\right)=0, \quad \lim _{n \rightarrow \infty} d\left(g F\left(y_{n}, x_{n}\right), F\left(g y_{n}, g x_{n}\right)\right)=0
$$

whenever $\left\{x_{n}\right\}$ and $\left\{y_{n}\right\}$ are the sequences in $X$ such that $\lim _{n \rightarrow \infty} F\left(x_{n}, y_{n}\right)=\lim _{n \rightarrow \infty} g x_{n}$ and $\lim _{n \rightarrow \infty} F\left(y_{n}, x_{n}\right)=\lim _{n \rightarrow \infty} g y_{n}$. 
Now, we prove some results to show how coupled notions (as the compatibility) can be reduced to the unidimensional case using the mappings defined as follows:

Let $X$ be a nonempty set and $F: X \times X \rightarrow X, g: X \rightarrow X$ be two mappings. Define two mappings $T_{F}^{2}, G^{2}: X \times X \rightarrow X \times X$ by

$$
T_{F}^{2}(x, y)=(F(x, y), F(y, x))
$$

and

$$
G^{2}(x, y)=(g x, g y)
$$

for all $x, y \in X$, respectively.

For instance, the following lemma guarantees that the 2-dimensional notion of common fixed coincidence points can be interpreted in terms of two mappings $T_{F}^{2}$ and $G^{2}$.

Lemma 3.4 Let $X$ be a nonempty set and $F: X \times X \rightarrow X$ and $g: X \rightarrow X$ be two mappings. Then s point $\left(x_{1}, x_{2}\right) \in X \times X$ is:

(1) a coupled fixed point of $F$ if and only if it is a fixed point of the mapping $T_{F}^{2}$;

(2) a coupled coincidence point of $F$ and $g$ if and only if it is a coincidence point of two mappings $T_{F}^{2}$ and $\mathrm{G}^{2}$;

(3) a coupled fixed point of $F$ and $g$ if and only if it is a common fixed point of two mappings $T_{F}^{2}$ and $G^{2}$.

Proof In order to avoid the repetition, we will only show the proof in the case of coupled fixed point. For any $\left(x_{1}, x_{2}\right) \in X \times X$, we obtain

$$
\begin{gathered}
\left(x_{1}, x_{2}\right) \in X^{2} \text { is a coupled fixed point of } F \\
\Longleftrightarrow F\left(x_{1}, x_{2}\right)=x_{1} \text { and } F\left(x_{2}, x_{1}\right)=x_{2} \\
\Longleftrightarrow \quad\left(F\left(x_{1}, x_{2}\right), F\left(x_{2}, x_{1}\right)\right)=\left(x_{1}, x_{2}\right) \\
\Longleftrightarrow T_{F}^{2}\left(x_{1}, x_{2}\right)=\left(x_{1}, x_{2}\right) \\
\Longleftrightarrow \quad\left(x_{1}, x_{2}\right) \text { is a fixed point of } T_{F}^{2} .
\end{gathered}
$$

Now, we show how to use Theorem 2.3 in order to deduce coupled fixed point results.

Theorem 3.5 Let $\left(X, d^{\prime}, \preceq\right)$ be a complete partially ordered metric space, $d$ be another metric on $X$ and $g: X \rightarrow X, F: X \times X \rightarrow X$ be two mappings such that $F$ has the $g$-monotone property. Suppose that the following conditions hold:

(1) $g:\left(X, d^{\prime}\right) \rightarrow\left(X, d^{\prime}\right)$ is continuous and $g(X)$ is $d^{\prime}$-closed;

(2) $F(X \times X) \subseteq g(X)$;

(3) there exist $x_{0}, y_{0} \in X$ such that $g x_{0} \preceq F\left(x_{0}, y_{0}\right)$ and $g y_{0} \preceq F\left(y_{0}, x_{0}\right)$;

(4) there exists $\theta \in \Theta$ such that

$$
d(F(x, y), F(u, v)) \leq \theta(\max \{d(g x, g u), d(g y, g v)\}) \max \{d(g x, g u), d(g y, g v)\}
$$

for all $x, y, u, v \in X$ with $g x \preceq g u$ and $g y \preceq g v$ or $g x \succeq g u$ and $g y \succeq g v$; 
(5) if $d \ngtr d^{\prime}$, assume that $F:(X, d) \times(X, d) \rightarrow\left(X, d^{\prime}\right)$ is g-uniformly continuous;

(6) if $d \neq d^{\prime}$, assume that $F:\left(X, d^{\prime}\right) \times\left(X, d^{\prime}\right) \rightarrow\left(X, d^{\prime}\right)$ is continuous and $g$ and $F$ are $d^{\prime}$-compatible;

(7) if $d=d^{\prime}$, assume that (a) $F$ is continuous and $g$ and $F$ are compatible or (b) for any non-decreasing sequence $\left\{x_{n}\right\}$ in $X$, if $x_{n} \rightarrow x \in X$ as $n \rightarrow \infty$, then $x_{n} \preceq x$ for all $n \geq 1$.

Then there exist $u, v \in X$ such that $g u=F(u, v)$ and $g v=F(v, u)$, i.e., $g$ and $F$ have a coupled coincidence point.

Proof It is only necessary to apply Theorem 2.3 to the mappings $T_{F}^{2}$ and $G^{2}$ in complete partially ordered metric space $\left(X \times X, D^{\prime}, \preceq\right)$ and metric space $(X \times X, D)$, where

$$
\begin{aligned}
& D^{\prime}((x, y),(u, v))=\max \left\{d^{\prime}(x, u), d^{\prime}(y, v)\right\}, \\
& D((x, y),(u, v))=\max \{d(x, u), d(y, v)\}
\end{aligned}
$$

and

$$
(x, y) \preceq(u, v) \Longleftrightarrow x \preceq u, y \preceq v
$$

for all $(x, y),(u, v) \in X \times X$. For example, the $D^{\prime}$-compatibility:

$F$ and $g$ are $d^{\prime}$-compatible

$$
\begin{gathered}
\Longleftrightarrow \quad\left[\begin{array}{l}
\lim _{n \rightarrow \infty} F\left(x_{n}, y_{n}\right)=\lim _{n \rightarrow \infty} g x_{n} \\
\lim _{n \rightarrow \infty} F\left(y_{n}, x_{n}\right)=\lim _{n \rightarrow \infty} g y_{n}
\end{array}\right\} \\
\left.\Longrightarrow \quad \begin{array}{l}
\lim _{n \rightarrow \infty} d^{\prime}\left(g F\left(x_{n}, y_{n}\right), F\left(g x_{n}, g y_{n}\right)=0\right. \\
\lim _{n \rightarrow \infty} d^{\prime}\left(g F\left(y_{n}, x_{n}\right), F\left(g y_{n}, g x_{n}\right)\right)=0
\end{array}\right] \\
\Longleftrightarrow \quad\left[\lim _{n \rightarrow \infty} T_{F}^{2}\left(x_{n}, y_{n}\right)=\lim _{n \rightarrow \infty} G^{2}\left(x_{n}, y_{n}\right)\right. \\
\left.\quad \Longrightarrow \quad \lim _{n \rightarrow \infty} D^{\prime}\left(T_{F}^{2} G^{2}\left(x_{n}, y_{n}\right), G^{2} T_{F}^{2}\left(x_{n}, y_{n}\right)\right)=0\right] \\
\Longleftrightarrow \quad T_{F}^{2} \text { and } G^{2} \text { are } D^{\prime} \text {-compatible. }
\end{gathered}
$$

This completes the proof.

Taking $d=d^{\prime}$ in Theorem 3.5, we get the following result in [13]:

Corollary 3.6 ([13], Theorem 3.1) Let $(X, d, \preceq)$ be a complete partially ordered metric space and $g: X \rightarrow X, F: X \times X \rightarrow X$ be two mappings such that $F$ has the $g$-monotone property. Suppose that the following hold:

(1) $g$ is continuous and $g(X)$ is closed;

(2) $F(X \times X) \subseteq g(X)$;

(3) there exist $x_{0}, y_{0} \in X$ such that $g x_{0} \preceq F\left(x_{0}, y_{0}\right)$ and $g y_{0} \preceq F\left(y_{0}, x_{0}\right)$;

(4) there exists $\theta \in \Theta$ such that

$$
d(F(x, y), F(u, v)) \leq \theta(\max \{d(g x, g u), d(g y, g v)\}) \max \{d(g x, g u), d(g y, g v)\}
$$

for all $x, y, u, v \in X$ with $g x \preceq g u$ and $g y \preceq g v$ or $g x \succeq g u$ and $g y \succeq g v$; 
(5) (a) $F$ is continuous and $g$ and $F$ are compatible or (b) for any non-decreasing sequence $\left\{x_{n}\right\}$ in $X$, if $x_{n} \rightarrow x \in X$ as $n \rightarrow \infty$, then $x_{n} \preceq x$ for all $n \geq 1$.

Then there exist $u, v \in X$ such that $g u=F(u, v)$ and $g v=F(v, u)$, i.e., $g$ and $F$ have a coupled coincidence point.

Definition 3.7 Let $(X, \preceq)$ be a partially ordered set and $F: X \times X \rightarrow X, g: X \rightarrow X$ be two mappings. The mapping $F$ is said to have the $g$-mixed monotone if $F$ is $g$-non-decreasing in its first argument and $g$-non-increasing in its second one, that is, for any $x, y \in X$,

$$
x_{1}, x_{2} \in X, \quad g x_{1} \preceq g x_{2} \quad \Longrightarrow \quad F\left(x_{1}, y\right) \preceq F\left(x_{2}, y\right)
$$

and

$$
y_{1}, y_{2} \in X, \quad g y_{1} \preceq g y_{2} \quad \Longrightarrow \quad F\left(x, y_{1}\right) \succeq F\left(x, y_{2}\right) .
$$

If, in the previous relations, $g$ is the identity mapping, then $F$ is said to have the mixed monotone property.

Now, we show how to use Theorem 2.3 in order to deduce coupled fixed point results with the $g$-mixed monotone properties.

Theorem 3.8 Let $\left(X, d^{\prime}, \preceq\right)$ be a complete partially ordered metric space, $d$ be another metric on $X$ and $g: X \rightarrow X, F: X \times X \rightarrow X$ be two mappings such that $F$ has the $g$-mixed monotone property. Suppose that the following hold:

(1) $g:\left(X, d^{\prime}\right) \rightarrow\left(X, d^{\prime}\right)$ is continuous and $g(X)$ is $d^{\prime}$-closed;

(2) $F(X \times X) \subseteq g(X)$;

(3) there exist $x_{0}, y_{0} \in X$ such that $g x_{0} \preceq F\left(x_{0}, y_{0}\right)$ and $g y_{0} \succeq F\left(y_{0}, x_{0}\right)$;

(4) there exists $\theta \in \Theta$ such that

$$
d(F(x, y), F(u, v)) \leq \theta\left(\frac{d(g x, g u)+d(g y, g v)}{2}\right) \frac{d(g x, g u)+d(g y, g v)}{2}
$$

for all $x, y, u, v \in X$ satisfying $g x \preceq g u$ and $g y \succeq g v$ or $g x \succeq g u$ and $g y \succeq g v$;

(5) if $d \ngtr d^{\prime}$, assume that $F:(X, d) \times(X, d) \rightarrow\left(X, d^{\prime}\right)$ is $g$-uniformly continuous;

(6) if $d \neq d^{\prime}$, assume that $F:\left(X, d^{\prime}\right) \times\left(X, d^{\prime}\right) \rightarrow\left(X, d^{\prime}\right)$ is continuous and $g$ and $F$ are $d^{\prime}$-compatible;

(7) if $d=d^{\prime}$, assume that (a) $F$ is continuous and $g$ and $F$ are compatible or (b1) for any non-decreasing sequence $\left\{x_{n}\right\}$ in $X$, if $x_{n} \rightarrow x \in X$ as $n \rightarrow \infty$, then $x_{n} \preceq x$ for all $n \in \mathbb{N}$ and (b2) for any non-increasing sequence $\left\{x_{n}\right\}$ in $X$, if $x_{n} \rightarrow x \in X$ as $n \rightarrow \infty$, then $x_{n} \succeq x$ for all $n \in \mathbb{N}$.

Then there exist $u, v \in X$ such that $g u=F(u, v)$ and $g v=F(v, u)$, i.e., $g$ and $F$ have a coupled coincidence point.

Proof It is only necessary to apply Theorem 2.3 to the mappings $T_{F}^{2}$ and $G^{2}$ in complete partially ordered metric space $\left(X \times X, D^{\prime}, \preceq\right)$ and metric space $(X \times X, D)$, where

$$
D^{\prime}((x, y),(u, v))=\frac{d^{\prime}(x, u)+d^{\prime}(y, v)}{2}
$$




$$
D((x, y),(u, v))=\frac{d(x, u)+d(y, v)}{2},
$$

and

$$
(x, y) \preceq(u, v) \quad \Longleftrightarrow \quad x \preceq u, y \succeq v
$$

for all $(x, y),(u, v) \in X \times X$. This completes the proof.

Remark 3.9 In the above result, if $g$ is the identity mapping and $d=d^{\prime}$, then we obtain Theorem 2.1 in [14].

\section{Competing interests}

The authors declare that they have no competing interests.

\section{Authors' contributions}

All authors contributed equally and significantly in writing this paper. All authors read and approved the final manuscript.

\section{Author details}

${ }^{1}$ Department of Mathematics, University of Jaén, Campus las Lagunillas s/n, Jaén, 23071, Spain. ²Department of Mathematics and Statistics, Faculty of Science and Technology, Thammasat University Rangsit Center, Pathumthani, 12121, Thailand. ' ${ }^{3}$ Department of Mathematics Education and the RINS, Gyeongsang National University, Chinju, 660-701, Korea. ${ }^{4}$ Department of Mathematics, King Abdulaziz University, Jeddah, 21589, Saudi Arabia.

\section{Acknowledgements}

The second author would like to thank the Thailand Research Fund and Thammasat University under Grant No. TRG5780013 for financial support during the preparation of this manuscript. Also, Yeol Je Cho was supported by Basic Science Research Program through the National Research Foundation of Korea (NRF) funded by the Ministry of Science, ICT and future Planning (2014R1A2A2A01002100).

\section{Received: 21 April 2015 Accepted: 14 September 2015 Published online: 26 September 2015}

\section{References}

1. Geraghty, M: On contractive mappings. Proc. Am. Math. Soc. 40, 604-608 (1973)

2. Amini-Harandi, A, Emami, H: A fixed point theorem for contraction type maps in partially ordered metric spaces and application to ordinary differential equations. Nonlinear Anal. 72, 2238-2242 (2010)

3. Eshaghi Gordji, M, Ramezani, M, Cho, YJ, Pirbavafa, S: A generalization of Geraghty's theorem in partially ordered metric spaces and application to ordinary differential equations. Fixed Point Theory Appl. 2012, 74 (2012)

4. La Rosa, V, Vetro, P: Fixed points for Geraghty-contractions in partial metric spaces. J. Nonlinear Sci. Appl. 7, 1-10 (2014)

5. Mongkolkeha, C, Cho, YJ, Kumam, P: Best proximity points for Geraghty's proximal contraction mappings. Fixed Point Theory Appl. 2013, 180 (2013)

6. Agarwal, RP, O'Regan, D: Fixed point theory for generalized contractions on spaces with two metrics. J. Math. Anal. Appl. 248, 402-414 (2000)

7. Maia, MG: Un'obsservazione sulle contrazioni metriche. Rend. Semin. Mat. Univ. Padova 40, 139-143 (1968)

8. Precup, R: Discrete continuation method for boundary value problems on bounded sets in Banach spaces. J. Comput. Appl. Math. 113, 267-281 (2000)

9. Jungck, G: Compatible mappings and common fixed points. Int. J. Math. Math. Sci. 9, 771-779 (1986)

10. Gnana Bhaskar, T, Lakshmikantham, V: Fixed point theorems in partially ordered metric spaces and applications. Nonlinear Anal. 65, 1379-1393 (2006)

11. Lakshmikantham, V, Ćirić, L: Coupled fixed point theorems for nonlinear contractions in partially ordered metric spaces. Nonlinear Anal. 70, 4341-4349 (2009)

12. Choudhury, BS, Kundu, A: A coupled coincidence point result in partially ordered metric spaces for compatible mappings. Nonlinear Anal. 73, 2524-2531 (2010)

13. Kadelburg, Z, Kumam, P, Radenovic, S, Sintunavarat, W: Common coupled fixed point theorems for Geraghty-type contraction mappings using monotone property. Fixed Point Theory Appl. 2015, 27 (2015)

14. Choudhurya, BS, Kundu, A: On coupled generalized Banach and Kannan type contractions. J. Nonlinear Sci. Appl. 5, 259-270 (2012) 